\title{
Research on importance of cleanliness in manufacturing reliable products for automotive
}

\author{
Alexandra Dzetzit*, and Gheorghe Nagit \\ "Gheorghe Asachi" Technical University of Iasi, Department of Machine \\ Manufacturing Technology, Romania
}

\begin{abstract}
Quality is ultimately defined by the client and is near the result of requirements and customer expectations. Currently the molten aluminum has known a huge increase in demands. Once with the enlarged volumes is also expected a high purity of the raw material which starts with the initial state of aluminum alloy. In the presented paper one of the methods that can assure the necessary quality is presented, with focus on particles and how this final result can be improved. What is even more important, in addition for the detection method is the effective and appropriate method of reducing them. The final results, the beneficial effects of filtering die casting metal with the bonded particle filter media, are presented in different aspects in order to achieve reduction of machining defects that in the ends achieves financial and casting marketability realizations.
\end{abstract}

\section{Introduction}

The automotive industries is growing fast and during the last year the technical development had inquired special classes for technical cleanliness. Nowadays, the cleanliness had become vital to the automotive "freedom of a control-or function area of critical impurities coming from the production process and environment". The problems with the product's cleanliness had always been there but now, once with the customer's growing demand for safety and comfort in driving, cleanliness it is necessaries to ensure quality and reliability in respect of the technical cleanliness standard [1]. Due to that, the tolerances of assemblies build into the vehicles are lower and lower and that for the parts are compiled to support higher and higher stress and therefore them are more sensitive to particle contamination. The standards are compiling to smaller classes and without regard to, imagine that a chip just a few microns in length can compromise an entire complex hydraulic system or even the completed fail of the system.

The particles can create the breakdown of diesel injection system than can reach the pressure of 2500 bars, a PCB (printed circuit board) that can cause an overstressed or short circuit, nozzles become blocked, valves congest, the bearing are damaged, this are only some of the reasons that can happen. In order to prevent the contamination, it is important to standardize the environmental production, for example the clean room's classes, particle monitoring, clothes, air conditioning. Development department is responsible for creating the

\footnotetext{
* Corresponding author : dzetzit.alexandra@yahoo.com
} 
product specification, since technical cleanliness is a design feature. In general, cleanliness specification for a part, component or product are given to secure the function and/or to fulfill a cosmetic specification. State of the art is to distinguish between 3 classes by a light microscope with polarization filter (one of the measuring methods): metallic shiny, nonmetallic shiny and fibers. Therefore, will be presented some methods for monitoring the inclusions inside the molten aluminum in the following paper, but in different aspects than concluding with the benefits that can be taken out of this method of measure. The system is capable of monitoring in raw material, the concentration and size of particles in the range of $20-300 \mu \mathrm{m}$ in the absence of micro bubbles in the melt of aluminum [3].

\section{Cleanliness determining tolerances and producing parts}

\subsection{Main ideas}

A cleanliness specification should be taken in account where there is a significant risk because the system's function or functionality of a component could be serious damaged by particles contamination. But, if this risk it is not present, a cleanliness specification should not be made. Cleanliness is a characteristic that should be taken an account from the first phase of the product- design. Before establishing cleanliness limiting values, it is necessary to check the automotive standard [1] and other standards, in order to increasing the compactability of the system. A good example could be the installation of filters, magnets, air filtration or a clean room and if it is established that the component has a safety reliability characteristic. Other methods for realizing this proactive, feasible and money saving is also the most convenient and safely, reducing the metallic chips and non-metallic into the manufacturing process [2].

\subsection{The importance of cleanliness classes in automotive}

Particles inside complex systems are generally mobile, this "weakest link in the chain" usually determines the degree of cleanliness necessary for the whole assembly. Examples of typical fluid systems found in the automotive industry include: the cooling system, the airconditioning and the exhaust system. A particle determined to be critical will only cause a malfunction if is located in the sensitive area of the system at the right moment in time and possibly if is orientated in a specific direction. For that the aim should be to determinate accurate the cleanliness standard in order to avoid:

-Damaging mechanisms/damaging effect (e.g. blocking, jamming, electrical shorting, etc.)

-Probability of a defect occurring, e.g. during a changeable state such as a valve closing;

-Large particle, high current lines - rapid corrosion

In Figure 1 are presented some possible failures from Jomesa showroom, failures produced by inclusions. This type of failures can cause leakages, blocker filters, blocking injectors, failures on pressure contacts or high contact resistance on electronic devices, bearing damages by hard particles, etc.

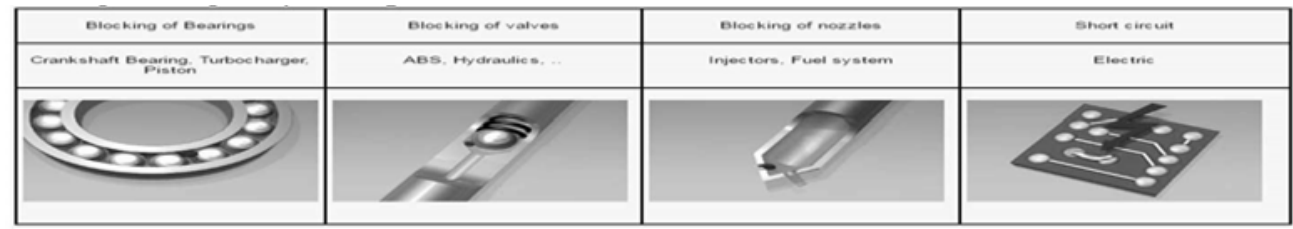

Fig. 1. Examples of defects from JOMESA microscope. [5] 


\section{Measuring cleanliness process}

Due to quick development and specially requirements from the customers, it is necessary to evolve and this has been driven by finding lightweight materials with strong mechanical qualities. For the moment, the most conceivable, but least productive method is to expect for the customer to accuse the part for an incident and in this matter to diagnose an issue. For sure, the best manner to handle the problem is not the one in question.

It is possible that the defect is not caught on time and creates an incident that could cause loss of human life. Maybe this is an exaggerate effect but the reality is the things can occur. In case of aluminum it could be said that one of the most important parameters is metal cleanliness defined like size and per solid inclusions per unit volume. That is why during the paper it will be discussed a method, maybe not new on the market, but continuously developed, using the history behind.

By this way, was created Limca III, for detecting inclusions in molten aluminum during processing. It is known that these particles can degrade the mechanical proprieties and also can damage the finished surfaces. Defects like small holes, tears, whiskers, visual defects on polished surfaces can occur. Casting defects that are related to molten metal cleanliness result from three main categories:

1.General inclusion level and oxide films present in the bulk liquid;

2.Local clusters of inclusions and oxide films caused by poor metal treatment;

3.Formation of oxide films during mold filling itself [3];

It is much known in the literature [3] that the first two problems caused many incidents among casting's suppliers. Due to the critical high quality demands, products like thin gauge sheet for body stock, foil or plates and designed parts with complicates bodies, make the presence of non-metallically and metallically particles to be detected and eliminated. There were developed several techniques based on pre-concentration / filtration step, followed by metallographic analysis such as the PoDFA from Alcan and the LAIS from Union Carbide, like K-Mold or Metal Vision with the aim of monitoring the concentration of particles in the melt and in the product [3]. Due to mentioned fact those methods take several days for getting any results and this means the involvement of resources that were required to study other solutions.

Besides, for this type of process procedure are mandatory the human resources with strong background on metallurgical process in order to complete all the analyses. The final results can have an extreme impact on the output, but cumulating everything the cost is not worthy enough, especially if is done on a regular frequency. Investigation on the market for building up filters during the pressure filtration test itself had been running. But the most effective and quite new on the market is the one who is cleaning the molten alloys by removing from the beginning the solid inclusions, an instrument named Limca III. Over the years, from the ' 80 until now, several Limca instruments were developed. In the raw material, in molten aluminum, the solid inclusions are mainly non-metallic compounds, such as oxides, borides, carbides, nitrides, chlorides, fluxing salt [3].

Limca instruments is based on the fact that this type of particles are not such eagerly conductive that the molten aluminum, mainly using resistive pulse / electric anticipating area technique (ESZ) [8]. A non-conducting glass pipeline overflowed with a $300 \mu \mathrm{m}$ hole and couple of tungsten electrodes, one inside the tube and one outside, are inundate the molten aluminum during measurement, like the figure below (Figure2).

The particles non-metallically have an electrical dynamism different from the fluid that they are included. In this way, they are compress into an electrically bumper capsule. Achieving to pass through the gap, the volume of the influencing fluid is dismissed causing the temporally shifting in the orifice of the electrical resistance.

The actual pick is observed as a voltage pulse. In this way, the pulses are intensified, 
counted and classified by their magnitude. By this route, it is provided an exact number (it is important to mention that is expressed in thousands of inclusions per kilogram of metal $(\mathrm{k} / \mathrm{kg})$ ) and also the size distribution of the particles. The inclusion recognition is here disturbed in the presence of micro bubbles due to their non-conducting performance. Effort was spent to separate non- metallic inclusions and gas bubbles, but no final solution is available to measure proximate degasser units [4].

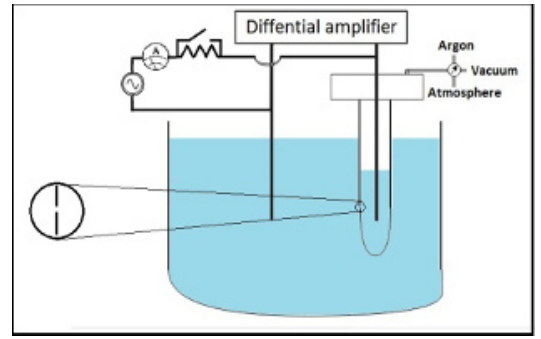

Fig. 2. Limca III functioning system.

In the latest research, it has been discovered that it was feasible ranging 20 microvolts signals, comparable to 20 microns inclusions in the molten aluminum during the process at $700^{\circ} \mathrm{C}$ in the power-driven rowdy climate of aluminum processing focal plant. The nonmetallic particles are detected in the molten aluminum due to limited-conductivity. This is realized by Limca using a vacuum to drag the molten aluminum into an aluminosilicate glass probe through 300 micron's loop. How also was described above, the electrical current is the same to all the opening [6].

Main difference between other methods and Limca III can be observed in the decisional tree (Figure 3.) and providing major improvements on casting process knowledge, with main influence on environment, productivity, recycling, flexibility, quality measurement.

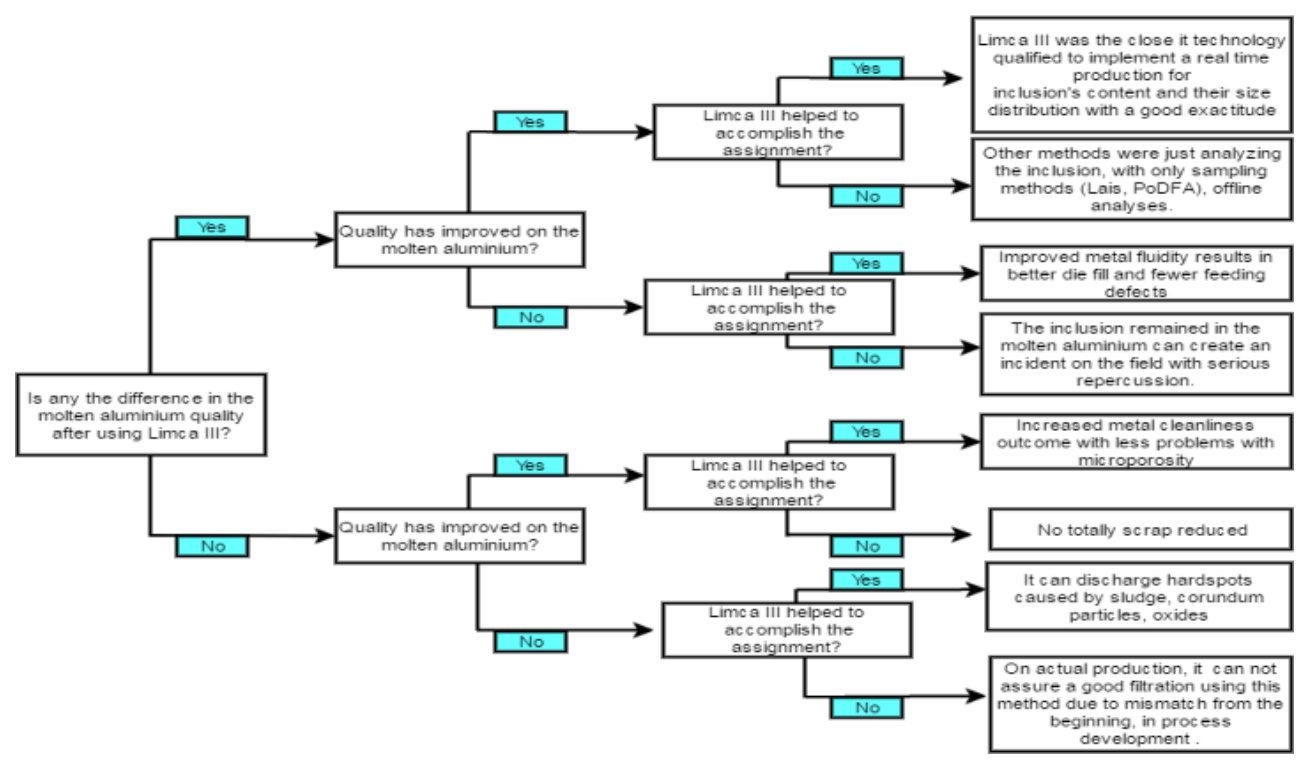

Fig. 3. Decisional tree in the benefits of using Limca. 


\section{Case study on Limca Ill's results}

Using all the terms involved in Figure 4, Isikawa diagram, the results can show the necessary step for introducing filtering particles inside molten aluminum (Figure 4).

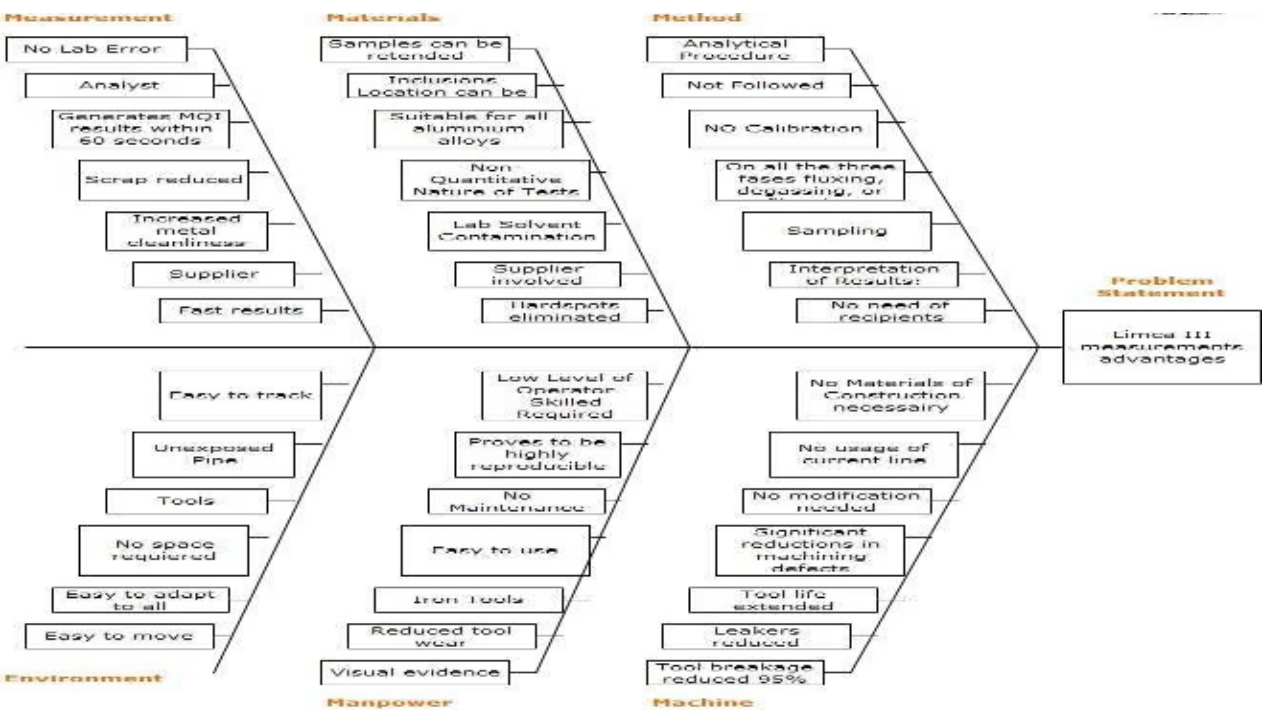

Fig. 4. Ishikawa- using Limca in the process line.

The results taken out from this measurement contain data that shown the actual cleanliness of the material used. Results are replicated but long-term metallurgical elements will depend on the standard procedures and starting stocks employed in the manufacturing process. In agreement with the length to which the material assessed in this study is exemplified, the inclusion content has a certain repeatability. In the following pictures, we can detect how distinct it is the process from the quantities detected. It can be seen in the Figure 5 that the values are from different stages of the casting: exit furnace, after degasser and CFF (ceramic foam filter). Background that had side effects on the measuring we can add: all tests were carried out at $685{ }^{\circ} \mathrm{C}$ and a pressure of $12.1 \mathrm{psi}$. The tests were carried out between 680 and $690^{\circ} \mathrm{C}$ and a pressure of 12.1 psi. The material used was A380 and the refined melt weight samples were $1000 \mathrm{~g}$ weight.

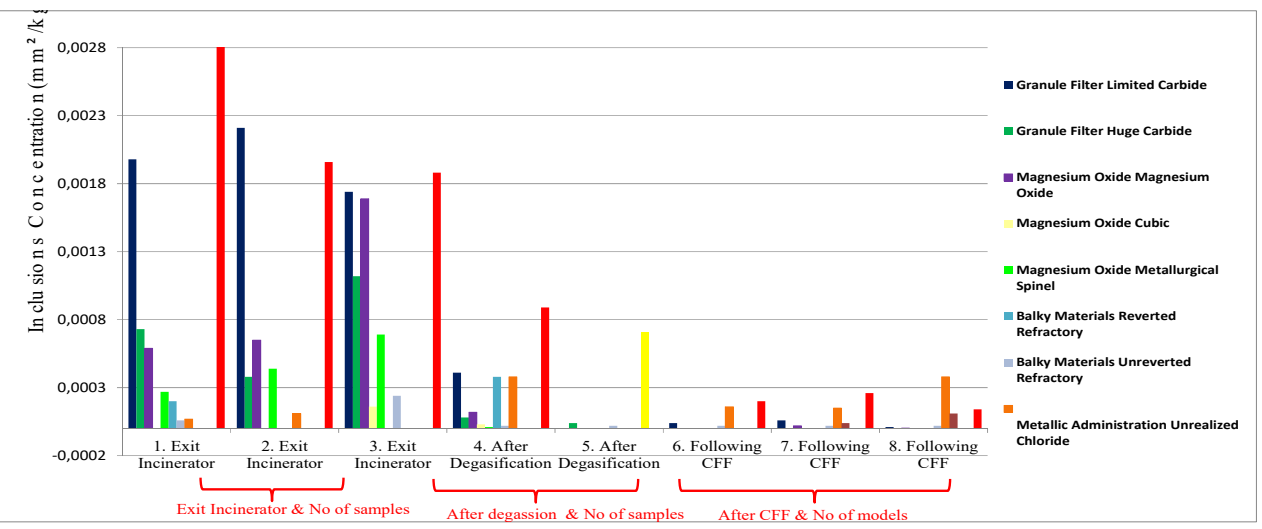

Fig. 5. Metallographic Analysis Results from a Limca measurement. 
Once the measurement is done, it is very visible in the graph below, (Figure 6), a significant improvement over unfiltered material and by this manner, the fluidity is improved with normal industry data.

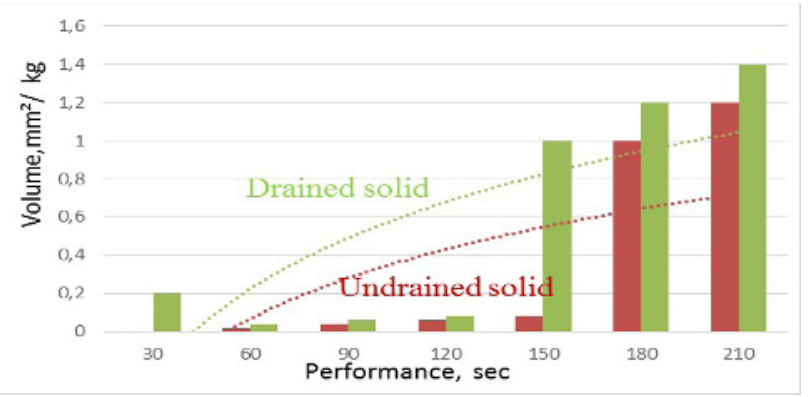

Fig. 6. Benefits of filtration of molten aluminum.

From the pictures, it can be seen that the metallurgical spinels (MgAl2O4) can cause big aversion in the aluminum process because of their large size and cohesion. The rate of structures increases as deliquesce is heating up. A hard glassy minerals consisting of an oxide of magnesium and aluminum formed in a cube-shape are generally formed from the reaction between $\mathrm{MgO}$ chunks and aluminum oxides [7]. Because of their large dimensions, both types of this formations can easily be dragging out of the metal. Most chloride inclusions derive from fluidity's equipment using chlorine gas as an inclusion hollow agent and in the end, is acting as a metal cleanliness process. Another process that is enlarging in time is that after downgrading in contact with magnesium in which alloys are included, the obstinate particles become similar in composition and color as a metallurgical formation, but due to their more increased size, they are easily detectable. Usually, refractory inclusions are large solid particles which may be exceptionally destructive to the practice.

\section{Conclusions}

By the end of this paper it can be seen that there is a clear difference between the quality of the metal in the melting and casting furnaces, as well as after the in-line treatment processes. Aluminum alloys and treatment applied on it, in order to meet the specification, especially in castings processes are significant in the cleaning process. Results are reproducible but longterm metallurgical quality will depend on the standard procedures and stocks built in the manufacturing processes. That is why utilizing Limca III has developed a new generation of treatment equipment in order to reduce and control the particles. Due to that, also the progress of consistent turnout specialty materials wouldn't be now in production without this continuous check on inclusion's dimension.

\section{References}

1. VDA 19, Inspection of Technical Cleanliness, 2nd (Revised Edition) (2014)

2. M. Rochowicz, M. Bargende, Intern. Stuttgarter Symposium, 641 (2015)

3. Alain A. Simard, The Minerals, Metals \& Material Society, 1090, (2000)

4. M. Badowski, S. Instone, The Minerals, Metals \& Materials Society,1071, (2012)

5. http://www.jomesa.com,

6. T. L. Buijs, D. Gagnon, C. Dupuis, The Minerals, Metals \& Materials Society, 973, (2015)

7. J. Smith, Metallographic Analyses, (Int. Al. Casting, Quebec, Canada, 2006)

8. R.I.L. Guthrie, Metallurgical and Material Transactions B, 31B, 767 (2000) 